\title{
Senescent Vision: Is It All The Fault Of The Lens?
}

\author{
R. A. WEALE \\ London
}

\begin{abstract}
Summary
Visual decrement correlated with age is often attributed to a progressive deterioration in the optical quality of the ocular media, notably the crystalline lens. Experimental evidence is presented to test the extent to which this hypothesis can be sustained. Recent data on lenticular scatter are shown to be useful in the visualisation and photography of cataract in man.
\end{abstract}

The conventional wisdom is that our vision deteriorates with age and that the reason for this is to be sought in an opacification and loss of optical homogeneity of our ocular media. In this respect the crystalline lens is said to be so paramount as to be a bête noire. What is the evidence and is the conclusion valid?

\section{(a) Visual acuity and contrast sensitivity}

A large number of workers have tackled the problem of the senescence of vision, ${ }^{1}$ with reference to the influence of such physical factors as the pupillary diameter, lenticular transmissivity, and the changes in retinal illumination consequent upon the ageing processes associated with these factors. It is noteworthy that clinical workers tend to set these influences aside, and to implicate lenticular discontinuities, bubbles, and manifestations of local changes in refractive index in order to account for the lower visual acuities of the patients presenting to them. They naturally see fewer of the healthy ones.

By contrast, research workers who strive to sample as normal a population as they may find tend to eschew pathological observers with the result that their picture of the senescent decrement is somewhat less pessimistic. In point of fact, visual acuity in a healthy population drops from its peak in the twenties and thirties only to 0.5 at the age of about 80 years. ${ }^{2,3}$

\section{(b) Clinical view of the normal lens}

The clinical view is supported by changes in the lens that are well-nigh universal, and observed in normal and otherwise healthy eyes. There are the various mechanical and morphological changes examined in commendable detail by Fisher. ${ }^{4}$ His data and those obtained, e.g. in Nordmann's laboratory ${ }^{5}$ show that the old idea of the senescent lens sclerosing is untenable. Nevertheless its performance can in no way be said to improve with age. Added to this mechanical modification there is one of appearance, which suggests a deterioration in optical efficiency but, again, represents an idea that cannot be sustained. Lenticular senescence is accompanied by a change in colour, namely yellowing ${ }^{1}$ owing to a marked rise in ultra-violet absorbance (Fig. 1).

This situation offers a rationale for what is really an extrapolation: because the ageing lens changes its mechanical properties and this leads to visual problems manifesting as presbyopia, because it yellows and some cataracts are yellow or brunescent, and because cataractous lenses reveal discontinuities, ergo the decrease in visual acuity will be attributable to a deteriorating crystalline lens. The 


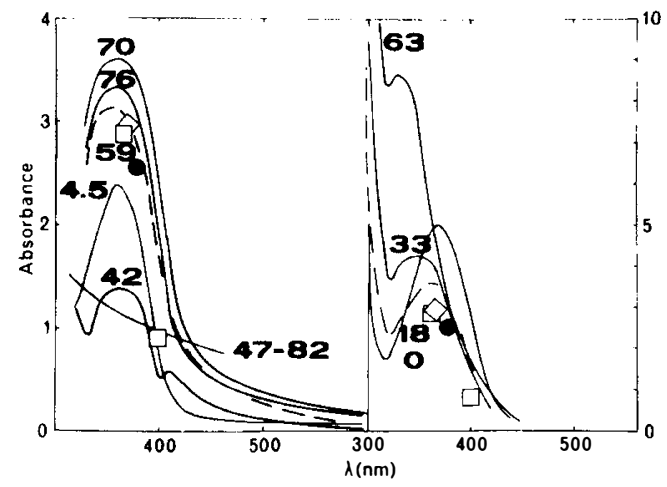

Fig. 1. Absorbance of crystalline lenses of various ages as a function of wavelength. Right-hand side (13); left-hand side: curve marked 47-82, ref. 15; other results, present author. Aphakic data: $\square,(19)$; O, (20); $\diamond,(21)$.

validity of this correlation is open to argument.

\section{(c) Lens colour and image formation}

In the first place, it is worth noting that a change in colour does not involve the ability of the lens to form a good retinal image if it is not accompanied by changes in internal refractive index: a black-and-white grating seen through a yellow lens may look black-and-yellow but it will not look fuzzy ${ }^{6}$. Real light scatter also leads to yellowing, and this will cause a reduction in apparent visual acuity: in this case, however, there are local variations in the lenticular refractive index. It follows that infor-
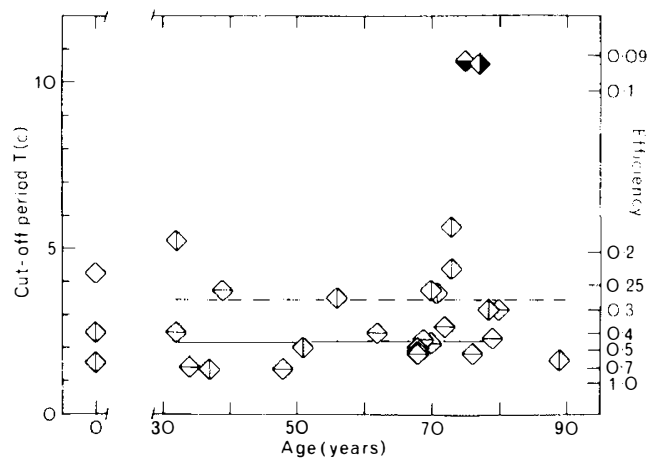

Fig. 2. Cut-off period (left ordinate) in cycles per minute of arc and efficiency (right ordinate) of male $(\theta)$ and female $(\bowtie)$ crystalline lenses as a function of age (abscissa). The half-black symbols refer to cloudy lenses. The full line is the regression for the male lenses, the broken one for the female ones (7). (Courtesy Academic Press) mation on lenticular colour will not in itself provide any information on whether or not the lens can form a good retinal image. Moreover, colour of the lens will, at most, indicate a predisposition for a reduced visual acuity: if intense it may reduce retinal illumination, a deficit that can be met by increasing illumination as long as the approximate homogeneity of the lens remains preserved.

These views are easily tested ${ }^{7}$. If an isolated crystalline lens is used to image gratings with a variety of bar-widths (spatial frequencies), it is possible to determine the finest grating which the lens will allow to be resolved, say, in a photographic record. Its frequency is called the cut-off frequency $f(c)$. This quantity can be calculated on a theoretical basis, and the ratio of the experimental and theoretical values of $f(c)$ is the efficiency of the lens. Figure 2 shows a plot of this value as a function of age: the data were obtained with approximately monochromatic (yellow) light, and those for women differ statistically from those for men. It will be noted that, over the range examined, there is no significant variation with age.

However, it is well known that, at shorter wave-lengths, the transmission factor decreases with advancing age, i.e. the absorbance rises. This has been measured by many authors, and an example is shown in Figure 3. There are some problems linked to this type of measurement which have been solved for better or for worse, a statement which cannot be made with the same degree of confidencemodest though it is-when it comes to the ultra-violet. When such light strikes a lens it is liable to fluoresce (e.g. ${ }^{8.9 .10}$ ): the radiation so produced may not vitiate the transmission measurements but can modify them. The amount of fluorescence increases with age (Fig. 4), and plays an adverse role, e.g. in the photography of cataracts the fluorescence of the nuclear type being well above normal. ${ }^{9}$

\section{(d) The ultra-violet part of the spectrum}

Although it has been realised for some two decades that the crystalline lens fulfils a role as a protective filter in so far as the vitreous humour and the retina are concerned, ${ }^{6.11}$ it is the recent boom in implants that has made it imperative to take the problem of ultra-violet 


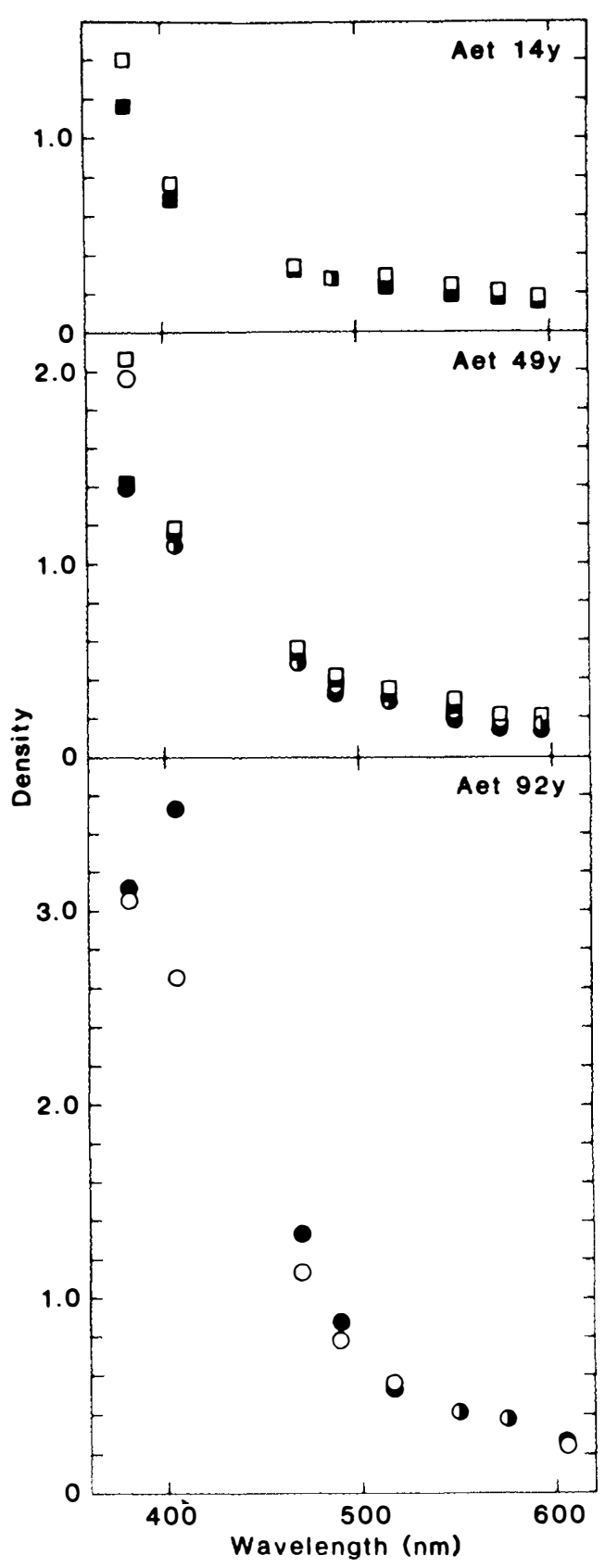

Fig. 3. Absorbance or photometric density (ordinate) as a function of wavelength (abscissa). The central section comprises data for two lenses of the same age. Male: $\bigcirc$, female: $\square$. The filled symbols represent measurements with a low intensity beam, the empty ones those obtained with a much more intense one to establish whether or not fluorescence affects the data (10). (Courtesy Academic Press) transmission seriously. Even so, the scientific literature is not flooded with unexceptionable results. The reliable data obtained by Boettner and Wolter ${ }^{12}$ are confined to a measurement on a single 4.5-year-old lens; Cooper and Robson ${ }^{13}$ used thin sections without taking into account the trauma such a procedure might inflict on the material with consequences for lens transmissivity that are only too apparent from the measurements; and Lerman and his collaborators ${ }^{14,15,16}$ obtained, over a decade, such a variability that it seems to have escaped their notice (Fig. 5).

None of these authors appears to have realised that their results can be compared with data obtained on unilateral aphakes whose ultra-violet visual threshold is measured in each eye in turn. The phakic eye will need more radiation to reach threshold than will its fellow in proportion as the intact crystalline lens absorbs more light than does the aphakic control. There is also the difficulty

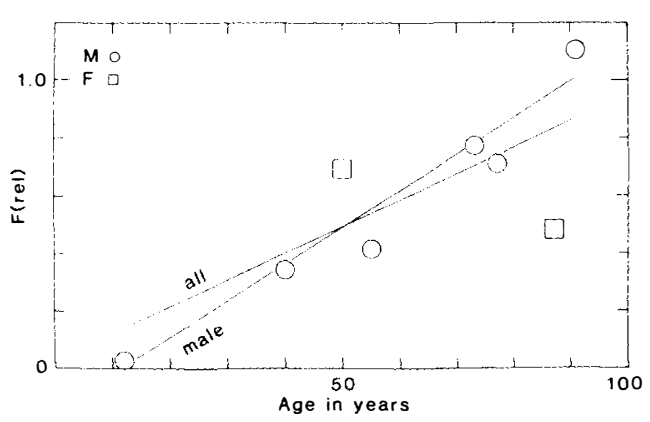

Fig. 4. Fluorescence in relative units (ordinate) as a function of age (abscissa). Male: $\bigcirc$; female: $\square$ (10). (Courtesy Academic Press)

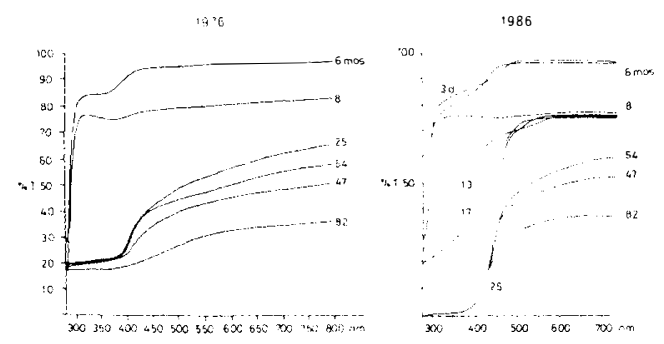

Fig. 5. Transmissivity (ordinate) of human crystalline lenses as a function of wavelength, with age as a parameter (after [14, 16]). Note the variability of the measurements in the ultra-violet part of the spectrum (after $[14,16])$. 

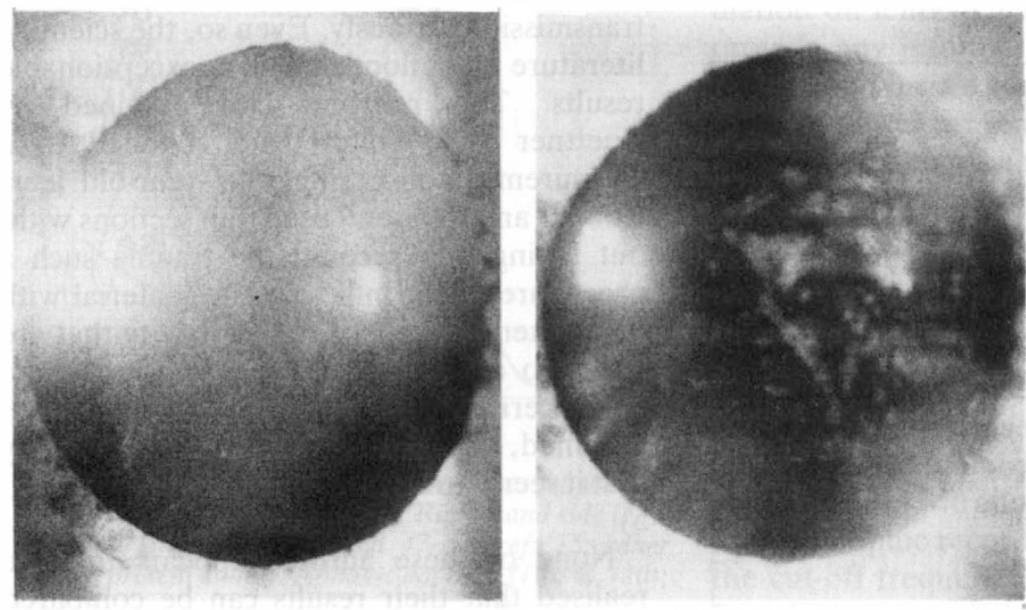

Fig. 6.

Comparison of $a$ cataractous lens in vivo, left: in conventional slitlamp illumination; right: in polarised and compensated light. of reliably measuring absorbances of more than 3 , i.e. transmissivities of less than 0.1 per cent. Figure 1 shows a comparison of data obtained by the above authors, together with some preliminary ones recorded by the author on a Perkin-Elmer spectrograph, great care having been taken with stray light. The comparisons are juxtaposed to sensory measurements on unilateral aphakes. It will be seen that the recent data are compatible with them, those due to Cooper and Robson ${ }^{13}$ with some, but those due to Lerman and his colleagues ${ }^{14,15,16}$ only with the greatest, difficulty.

\section{(e) Lenticular scatter of light}

The slit-lamp reveals the lens as a source of considerable light scatter notably in the second half of the human life span. Linked to this general observation there is a well-known paradox that the patient's visual acuity is usually appreciably better than one's clinical experience of the slit-lamp appearance of his crystalline lens would allow one to expect.

Recent experiments provide an explanation of this paradox. When a slit-lamp was provided with a polariser and an analyser, and the birefringence of the ocular media was brought under control by means of a quarter-wave plate, a considerable reduction in the apparent scatter was observed ${ }^{17}$. The irreducible part of the scatter increased systematically with age, but its percentage of the total did not approach 100 much before the tenth decade of life. The light-scatter observed in the slit-lamp probably consists of two parts. On the one hand there are small variations in refractive index and fibre density which give rise to a compensatable phase difference between the two orthogonal components of light into which any polarised beam of light can be resolved. The use of the quarter-wave plate effects compensation with the result that the apparent scatter is reduced. The remainder is due, in all probability, to variations in optical density which cannot be eliminated in the above manner and may therefore be referred to as real light-scatter as distinct from the apparent variety.

\section{(f) Photography of the lens and cataracts}

The above observation applies not only to normal, healthy lenses but can be made also on the vast majority of cataractous ones. But this implies that, if opacifications and inhomogeneities which interfere with vision appear against a background made up of real and apparent scatter, the removal of the latter is likely to increase the contrast with which the photic obstacles can be visualised and photographed. Figure 6 shows that this is, in fact, true ${ }^{18}$.

There are admittedly exceptions to this. In a few cases of very advanced cataract (e.g. when the pupil appears white) the improvement in detail has failed to materialise. However, in such circumstances one is unlikely to be concerned with monitoring the progress of the problem. In cases of brunescent cataract, finer detail is also relatively rarely observed. 
But in most of the other cases the reverse is true.

This raises the question whether 'fogging' is not really a relatively late event in the aetiology of some types of cataract, and whether the basic insult is not rather one of morphological change and structure. If this should prove to be the case, then our present preoccupation with high-molecular-weight agglomerates may relate not to the aetiology of the relevant cataracts but to one of the sequelae of the disease process. The early events involve frequently the refractive index and not light scatter: it is significant that when the patients' visual acuity is carefully tested with a Landoldt- $\mathrm{C}$ and by informed staff, the fact that they can resolve gaps in some orientations but fail in other fixed ones cannot escape attention.

\section{(g) Conclusion}

In the light of the above results on the lens and of those obtained in some crucial studies on contrast sensitivity ${ }^{3}$ it would seem that one can give a tentatively negative reply to the question asked in the title of this paper. There is no denying that, in some cases, the lens must be held responsible for a reduction in visual acuity, but this cannot be considered to be the rule. It is probable that cell-death, no matter whether due to stochastic or photic causes, plays a dominant role. It is observed in the retina and in the central nervous system in general. In the absence of any repair processes being discovered or introduced, it is likely that this rather than the optics of the visual system will impose limits on the visual function of the senescent populations of the world.

\section{References}

${ }^{1}$ Weale RA: A biography of the eye: development, growth, age. London: HK Lewis 1982.

2 Weale RA: Senile changes in visual acuity. Trans. ophthalmol. Soc. UK 1975; 95: 36-8.
${ }^{3}$ Morrison JD, Mcgrath C: Assessment of the optical contributions to the age-related deterioration in vision. Quart. J. exp. physiol. 1985; 70: 249-69.

${ }^{4}$ Fisher RF: Presbyopia and the changes with age in the human crystalline lens. J. Physiol. (Lond.) 1973; 228: 765-79.

${ }^{5}$ Nordmann J: Le noyau du cristallin. I. la teneur en eau. Arch. ophtal. Paris 1973; 33: 81-6.

${ }^{6}$ Weale RA: In: The human lens in relation to cataract. p. 205. Ciba Foundation symposium 19. Amsterdam, Elsevier-Excerpta Medica-North Holland; 1973.

${ }^{7}$ Weale RA: Transparency and power of post-mortem human lenses: variation with age and sex. Exp. eye res. 1983 ; 36: 731-41.

8 Vogt A: Lehrbuch und Atlas der Spaltlampenmikroskopie des lebenden Auges. Vol. II. Berlin, Julius Springer; 1931.

${ }^{9}$ Lerman S, Yamanashi BS, Borkman RF, Saker E: Photoacoustic, fluorescence and light transmission spectra of normal, aging and cataractous lenses. Ophthalm. res. 1978; 10: 168-76.

${ }^{10}$ Weale RA: Human lenticular fluorescence and transmissivity and their effect on vision. Exp. eye res. 1985, 41: 457-73.

${ }^{11}$ Weale RA: Chorio-retinal hazards in aphakia. Br. J. Ophthalmol. 1971, 55: 853.

12 Boettner EA, Wolter JR: Transmission of the ocular media. Invest. Ophthalmol. 1962; 1: 776-82.

${ }^{13}$ Cooper GF, Robson JG: The yellow colour of the lens of man and other primates. J. Physiol. (Lond.) 1969; 203: 411-17.

${ }^{14}$ Lerman S, Borkman R: Spectroscopic evaluation and classification of the normal, aging, and cataractous lens. Ophthal. Res. 1976; 8: 335-53.

${ }^{15}$ Lerman S: Biophysical aspects of corneal and lenticular transparency. Curr. eye res. 1984; 3: 3-14.

${ }^{16}$ Lerman S: In vivo and in vitro biophysical studies on human cataractogenesis. Lens Res. 1986, 3: 13760.

17 Weale RA: Real light scatter in the human crystalline lens. Graefe's Arch. Clin. Exp. Ophthal. 1986; 224: 463-66.

18 Weale RA: A new method for visualising discontinuities in the crystalline lens. $\mathrm{Br} . \mathrm{J}$. Ophthalmol. 1986 (in press).

${ }^{19}$ Wald G: The photochemistry of vision. Doc. Ophthalmol. 1949; 3: 94-137.

${ }^{20}$ Gayton AG: Colour sensations produced by ultraviolet light. Proc. Phys. Soc. 1938; 50: 714-20.

${ }^{21}$ Werner JS, Hardenbergh E: Spectral sensitivity of the pseudophakic eye. Arch Ophthalmol. 1983; 101: 758-60. 